\title{
Loss of MTAP expression is a negative prognostic marker in Ewing sarcoma family of tumors
}

\author{
Lucas Faria Abrahao-Machado*1, Bruno Antunes ${ }^{2}$, Renee Zon Filippi ${ }^{3}$, Sahlua Volc ${ }^{4}$, Erica \\ Boldrini $^{5}$, Weder P Menezes ${ }^{6}$, Rui M Reis ${ }^{6,7,8}$ \& Olavo Pires de Camargo ${ }^{3}$ \\ ${ }^{1}$ Department of Pathology \& Molecular Diagnosis, Barretos Cancer Hospital, Barretos, SP, Brazil \\ ${ }^{2}$ Department of Othopedics Surgery, Barretos Cancer Hospital, Barretos, SP, Brazil \\ ${ }^{3}$ Institute of Orthopedics \& Traumatology, Faculty of Medicine, University of São Paulo (FMUSP), São Paulo, SP, Brazil \\ ${ }^{4}$ Oncology Department, Barretos Cancer Hospital, Barretos, SP, Brazil \\ ${ }^{5}$ Pediatrics Department, Barretos Cancer Hospital, Barretos, SP, Brazil \\ ${ }^{6}$ Molecular Oncology Research Center, Barretos Cancer Hospital, Barretos, SP, Brazil \\ ${ }^{7}$ Life \& Health Sciences Research Institute (ICVS), Health Sciences School, University of Minho, Braga, Portugal \\ ${ }^{8}$ ICVS/3B's-PT Government Associate Laboratory, Braga/Guimarães, Portugal \\ * Author for correspondence: Tel.: +55 14 31125956; Fax: +55 14 31125900; lucasfariamachado@gmail.com
}

\begin{abstract}
Aim: The Ewing sarcoma family of tumors (ESFT) is a group of malignant small round cell neoplasms of bones and soft tissues closely histogenetically related. Methylthioadenosine phosphorylase (MTAP) deficiency has been recently associated with increased tumor aggressiveness and poor outcomes in different types of neoplasms. However, the expression of this biomarker and its biological role in ESFT remain largely unknown. Methods: Immunohistochemical expression of MTAP was accessed in 112 patients with ESFT in a tissue microarray platform and associated with clinicopathological parameters and overall survival (OS). Results: Loss of MTAP expression was significantly associated with lower OS in both univariate and multivariate analyses. Conclusion: Loss of MTAP expression is an independent negative prognostic biomarker in ESFT.
\end{abstract}

First draft submitted: 3 May 2017; Accepted for publication: 26 September 2017; Published online: 15 December 2017

Keywords: biomarkers $\bullet$ ESFT $\bullet$ Ewing sarcoma family of tumors $\bullet$ methylthioadenosine phosphorylase $\bullet$ MTAP $\bullet$ prognosis

Methylthioadenosine phosphorylase (MTAP) is a protein product of the tumor suppressor gene located in the chromosome $9 \mathrm{p} 21$ region, close to the $p 16$ gene (CDKN2), which plays an important role in the metabolism of purines and polyamines [1]. In normal cells, MTAP cleaves MTA, a by-product of polyamine biosynthesis, into adenine and MTR-1-P. In MTAP-deficient cells, however, the MTA is not cleaved and the salvage pathway for adenine and methionine is absent, causing a stimulus for the de novo synthesis of purines [2].

Deficiency of this enzyme has been recently found in different types of neoplasms, mainly due to deletion of the 9p21 locus, and associated with increased tumor aggressiveness [1,3-5]. Su et al. (2014), in an immunohistochemistry (IHC) study of 99 lung cancer samples, demonstrated that MTAP was an independent marker of prognosis in patients with non-small-cell lung cancer [3]. In the study by Becker et al. (2015), the authors suggested that loss of MTAP expression may also be associated with increased malignancy of brain gliomas [5]. Similarly, De Oliveira et al. (2016), in a recent breast cancer study, found that loss of MTAP expression was associated with more aggressive tumor subtypes [4]. One of the possible explanations for the association with tumor aggressiveness is that in neoplastic cells MTAP inactivation stimulates de novo synthesis of purines, which is related to increased cell proliferation [5]. In contrast, MTAP-deficient neoplastic cells are more sensitive to purine synthesis inhibitor agents, such as methotrexate, azathioprine and $5^{\prime}$-fluorouracil $\left(5^{\prime}-\mathrm{FU}\right)$, which indicates that MTAP is a promising therapeutic target [2]. However, to date there are no reports on the IHC expression of MTAP in Ewing sarcoma family of tumors (ESFT). 
The ESFT comprises a spectrum of malignant neoplasms, consisting of small round cells with varying degrees of neuroectodermal differentiation, including bone and extraskeletal Ewing sarcoma, the so-called primitive neuroectodermal tumor (PNET) and Askin tumor [6]. These tumors are characterized by translocations involving one of the genes of the TET/FET family and one of the genes of the ETS family, most commonly EWSR1 and FLII $[\mathrm{t}(11,22)$ ( $\mathrm{q} 24 ; \mathrm{q} 12)]$, generating an aberrant transcription factor that can activate or repress other genes involved in the oncogenesis [7].

Over the last decades the therapeutic advances have brought a positive impact in reducing mortality of the disease, however the treatment of patients with localized or disseminated disease is still practically the same. That is, the differences between individuals and the biological differences between tumors are currently being ignored. Therefore, it is important to identify the tumors that may require different therapeutic interventions, especially those that can be more aggressive, with a greater chance of early metastasis and frequent relapses. The broader knowledge of the biology of ESFT and the interaction with each patient may contribute to the emergence of new personalized therapeutic modalities, especially molecular targeted therapies and, in this context, IHC also plays a relevant role. The immunohistochemical method is a widely and routinely used technique in most pathology laboratories. Much of the molecular findings of diseases, especially neoplasms, are validated or even originated from the evaluation of protein expression by IHC.

The aim of the present study was to evaluate the expression of MTAP by IHC and assess its clinicopathological impact in a well-characterized series of ESFT in a Brazilian population, in order to determine the value of this biomarker as a prognostic factor.

\section{Materials \& methods}

\section{Patients \& tumor samples}

Tumor samples from 112 patients with diagnosis of ESFT were retrieved from the Pathology Department of the Barretos Cancer Hospital, Brazil. All patients were diagnosed between 2001 and 2015 and the samples were taken from primary tumor biopsies prior to the use of any possible systemic treatment.

The diagnoses were confirmed by two independent expert pathologists of bone/soft tissue tumors, using all the methods currently used in routine pathology for diagnosis of ESFT, such as IHC, FISH and PCR. New IHC reactions, including CD99, Fli1 and NKX2.2 were used to confirm the diagnosis in all the reviewed cases, whereas FISH or PCR was applied in 67 out of 112 cases due to the paucity of tumor cells in many biopsy samples. The patients were grouped according to gender, age $(<20$ and $\geq 20$ years old), ethnicity (white and non-white), tumor location (axial vs peripheral and bone $v s$ soft tissues) and disease stage at diagnosis (localized and disseminated).

The study was conducted following national and institutional ethical policies and it was previously approved by the Barretos Cancer Hospital Ethical Committee (protocol 935/2015). Since it was classified as having minimal risk, ensuring confidentiality and not resulting in any clinical influences for the patients, the ethical committee waived the need for consent in this study.

\section{Tissue microarray construction}

After review of the cases and in case of sufficient tissue availability, the tumor areas of the respective paraffin blocks were marked for subsequent extraction and construction of tissue microarray (TMA). The TMAs were assembled using the Tissue Arrayer MTA-1 platform (Beecher Instruments, Inc., WI, USA). To represent possible heterogeneity of tumors and to avoid material losses due to wear or detachment, two 1.0-mm cores from each case were used in two TMA blocks. Non-neoplastic tissue was also added in the TMA blocks for use as positive controls. The TMAs were cut at $4.0 \mu$ in the microtome and their sections were stained with hematoxylin and eosin for histological re-examination and for subsequent immunohistochemical reactions.

\section{Immunohistochemical analysis}

A manual immunohistochemical assay on the TMA slides was performed for subsequent MTAP evaluation as previous reported [5]. Starfrost ${ }^{\circledR}$ adhesive slides (Kinttel, Braunshweig, Germany) were used for the immunohistochemical study. The slides were submitted to dewaxing and hydration by means of sequential baths of xylol and ethyl alcohol. Briefly, following sample dehydration and antigen retrieval for $4 \mathrm{~min}$ at $125^{\circ} \mathrm{C}$ and $20 \mathrm{~min}$ at $90^{\circ} \mathrm{C}$ with a mixed buffer (citrate $10 \mathrm{mM} /$ EDTA $1 \mathrm{mM}, \mathrm{pH}$ 6.0) the slides were incubated with the MTAP polyclonal antibody (Proteintech Group, Inc., cat 11475-1-AP) at a 1:300 dilution at room temperature overnight. The slides 


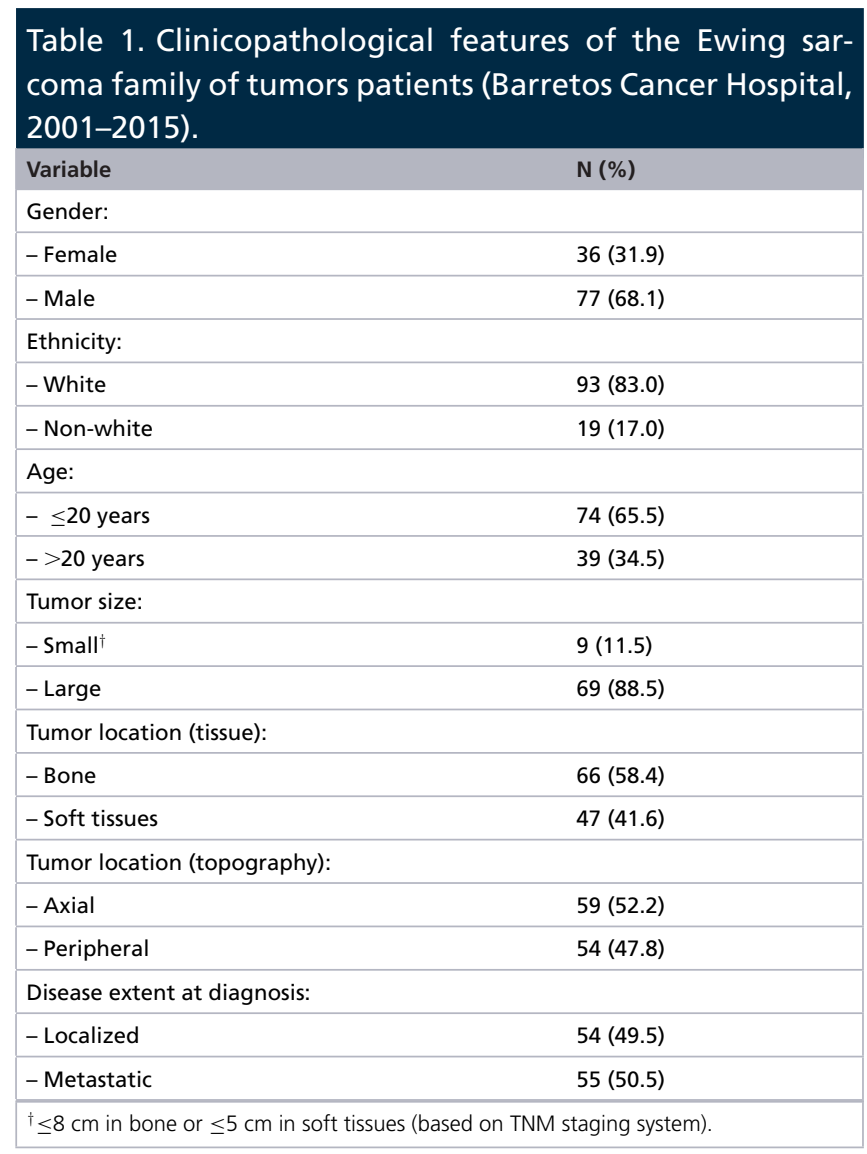

were then incubated with secondary antibody and streptavidin peroxidase, stained with $3,3^{\prime}$ diaminobenzidine chromogen and finally counterstained with hematoxylin.

The intensity and extension of MTAP immunoreactivity in the cytoplasm and/or nucleous were scored. Intensity of immunoreactivity was measured as: 0 (no staining); 1 (weak staining); 2 (moderate staining) and 3 (strong staining). The extension of immunoreactivity was assessed as: 0 (negative), 1 ( $\leq 25 \%$ of positive cells), 2 ( $25-50 \%$ of positive cells); or 3 ( $\geq 50 \%$ of positive cells). The sum of both parameters resulted in scores that finally classified the reactions as loss of expression (0-2) or retained expression (3-6).

\section{Statistical analysis}

The statistical analysis was performed using SPSS version 21.0 for Windows ${ }^{\mathrm{TM}}$ (IBM, IL, USA). For all variables, including clinical demographic characteristics and results of immunohistochemical reactions, frequencies and percentages were calculated. Associations between MTAP expression and the patient's characteristics were performed using the Pearson $\chi 2$ test or Fisher's exact test, depending on the expected values in the contingency tables. The overall survival (OS) analysis was done by constructing Kaplan-Meier curves and for a comparison between the obtained curves, the log-rank test was applied. An additional analysis of $O S$ was done by separating the patients into two groups: metastatic and nonmetastatic. OS was defined as the time between the date of diagnosis and the date of the last patient's information or death for any reason. In order to adjust for multiple prognostic factors, including the clinical parameters, and estimate the independent effect of MTAP expression on survival, a multivariate analysis was applied by the Cox regression method.

\section{Results}

The clinicopathological features of the ESFT patients \& associations with MTAP expression

The clinicopathological features of ESFT cases are summarized in Table 1. One-, 3- and 5-years OS were 69.9, 46 and $30.3 \%$, respectively. The male:female ratio in this study was $2.1: 1$. The majority of patients were white (83\%) and 20 years old or younger (65.5\%). Patient's age ranged from 2 to 67 years, with a mean of 19 years (standard 


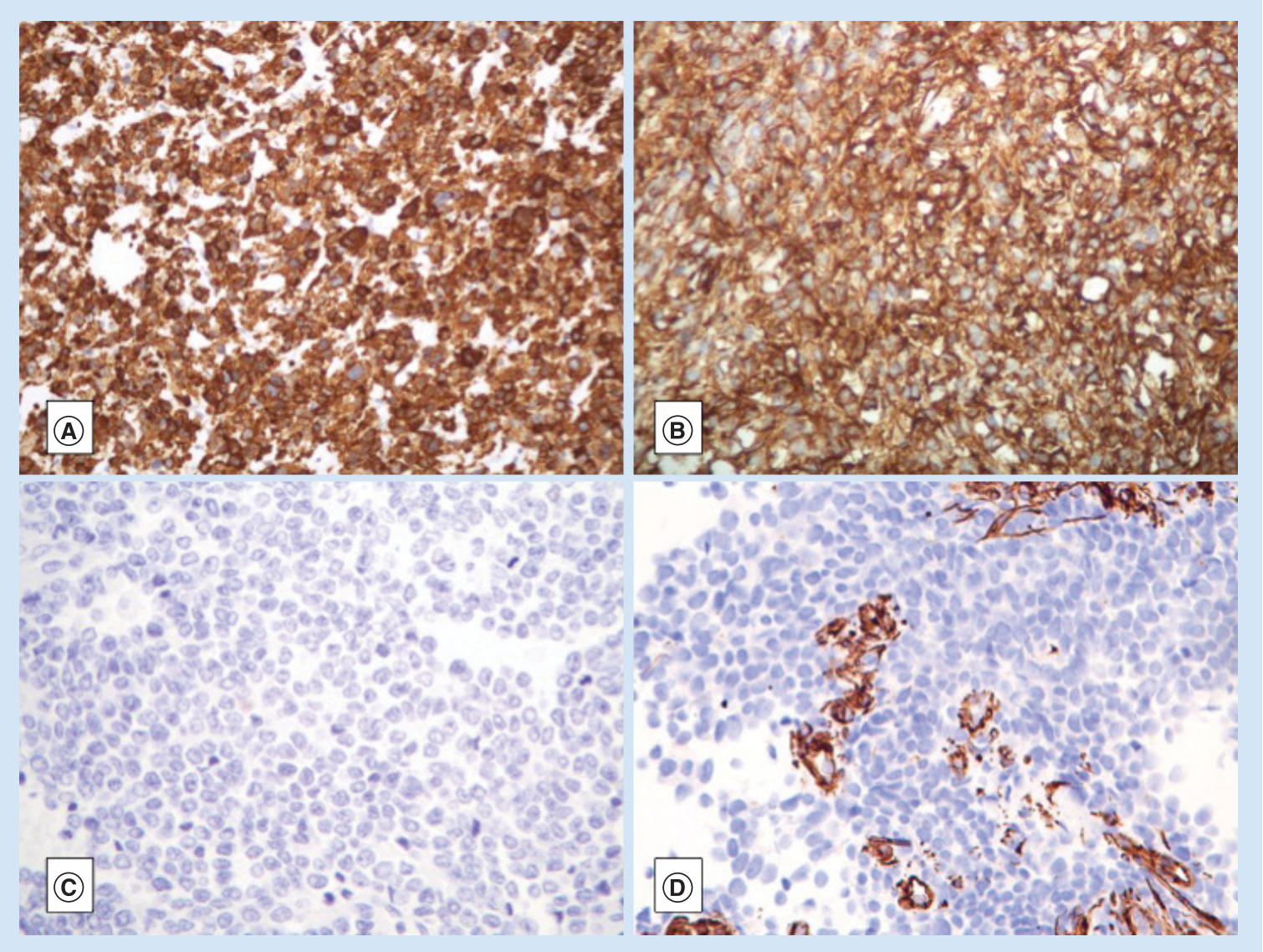

Figure 1 Methylthioadenosine phosphorylase immunohistochemistry expression. Positive cytoplasmic expression was observed in the majority of cases (A \& B) $400 \times$. (C \& D) $400 \times$ are examples of cases with total loss of MTAP expression in tumor cells. In (D) positive endothelial cells were also observed and served as internal controls for the reaction.

deviation: 12) and a median of 17 years (Q1: 12 and Q3: 21). Tumor dimensions ranged from 3.3 to $35.0 \mathrm{~cm}$, with a mean of $12.2 \mathrm{~cm}$ (standard deviation: 5.8) and a median of $11.0 \mathrm{~cm}$ (Q1: 8.5 and Q3: 14.5). A large proportion $(50.5 \%)$ of patients with metastasis at diagnosis was observed in this series.

Adequate IHC reactions were achieved in 111/112 of the cases. The IHC revealed cytoplasmic MTAP expression in 80/111 tumors (72.1\%; Figure 1A \& B). Thirty-one cases (27.9\%) presented loss of MTAP expression (Figure 1C $\& \mathrm{D})$.

We observed that MTAP expression profile was not significantly associated with the clinicopathological characteristics evaluated in the ESFT patients (Table 2).

\section{Association of MTAP expression with ESFT patients' prognosis}

MTAP expression presented a statistically significant association with OS, according to the Kaplan-Meier and log-rank methods $(\mathrm{p}=0.028$; Figure 2$)$. Patients with loss of MTAP expression had a 5-year OS rate of only $11.5 \%$, compared with $37.2 \%$ of patients with positive MTAP expression (Table 3 ). When patients were separated into two groups, metastatic and nonmetastatic, MTAP showed a statistically significant association with OS in the group of patients with metastasis $(\mathrm{p}=0.021)$. In the metastatic group, patients with loss of MTAP expression had a 3-year OS rate of only $16.7 \%$, compared with $35.0 \%$ of patients with positive MTAP expression. Regarding the other evaluated clinical characteristics, our results showed that non-white ethnicity $(\mathrm{p}=0.002)$, age over 20 years $(\mathrm{p}$ $=0,023)$ and metastatic disease at diagnosis $(\mathrm{p}=0,003)$ significantly influenced the OS (Table 3 ). The multivariate analysis, by the COX regression method, revealed that only loss of MTAP expression, ethnicity and metastasis at diagnosis were independent prognostic factors (Table 4). Regarding the metastatic group and non-metastatic group 


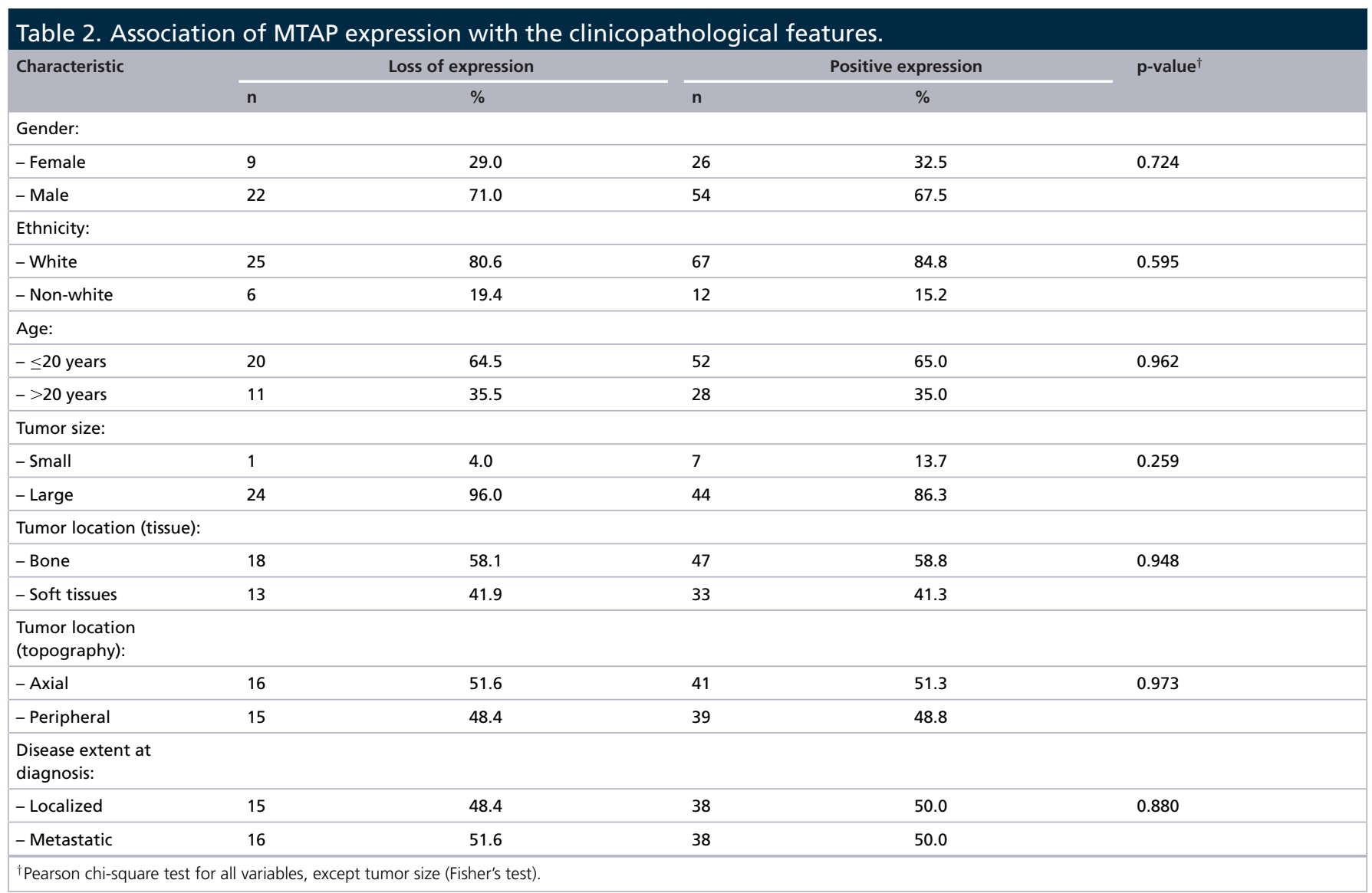

of patients, the multivariate analysis also showed a statistically significant association of MTAP loss with poorer OS in the metastatic group (Table 5).

\section{Discussion}

Although ESFT are high-grade sarcomas with a high metastasis rate, a significant increase in survival rates has been observed in the last few decades with the use of a multimodal therapeutic approach that includes chemotherapy, surgery and radiotherapy $[8,9]$. On the other hand, the survival rates for patients with metastatic or recurrent ESFT did not improve significantly, despite aggressive treatment [8-11].

The characterization of this Brazilian series of ESFT showed that more than half $(50.5 \%)$ of the patients $(n=54)$ had metastatic disease at diagnosis. This frequency is substantially higher than that observed in the literature, which typically describes the frequency of cases with metastasis at diagnosis around 25\% [6,8,9,12]. However, Brunetto et al. (2015) [10] had already demonstrated a significantly higher proportion of patients with metastatic disease in a Brazilian population, suggesting that this characteristic could reflect an association of multiple factors related to access and efficiency of the health system in Brazil. Barretos Cancer Hospital (BCH) is one of the largest cancer hospitals in Latin America, treating patients from all Brazilian states with $100 \%$ free services, covered by the National Health System of Brazil (SUS), and therefore, a reference bias for receiving more advanced cases must be considered. Although socioeconomic status was not a variable exploited in this study, the possibility of this factor influencing the frequency of cases with metastatic disease at diagnosis cannot be ruled out, just as demonstrated by Lee J et al. (2010) [12]. Many patients treated at BCH are very poor people and have passed through smaller and less specialized centers in their regions, being admitted after a long delay in diagnosis and initiation of treatment [13]. The extent of the disease at diagnosis is the most consistent prognostic factor according to the literature $[8-10,12,14]$. The results of the present study were consistent with previous studies, with a significantly lower OS in patients with metastatic disease, as expected. Multivariate analysis confirmed metastatic disease at diagnosis as an independent prognostic factor in our series of ESFT $(\mathrm{p}=0.002)$. 
Research Article Abrahao-Machado, Antunes, Filippi et al.

Table 3. Association of MTAP expression and clinicopathological features with overall survival. ${ }^{\dagger}$

\begin{tabular}{|c|c|c|c|c|c|c|}
\hline \multirow[t]{2}{*}{ Characteristic } & \multirow[t]{2}{*}{ Number of cases } & \multirow[t]{2}{*}{ Number of deaths } & \multicolumn{3}{|c|}{ OS (\%) } & \multirow[t]{2}{*}{ p-value } \\
\hline & & & 1 year & 3 years & 5 years & \\
\hline \multicolumn{7}{|l|}{ MTAP: } \\
\hline - Loss of expression & 31 & 24 & $58.1 \%$ & $37.3 \%$ & $11.5 \%$ & 0.028 \\
\hline - Positive expression & 80 & 46 & $75.0 \%$ & $50.7 \%$ & $37.2 \%$ & \\
\hline \multicolumn{7}{|l|}{ Gender: } \\
\hline - Female & 36 & 20 & $75.0 \%$ & $49.3 \%$ & $36.1 \%$ & 0.476 \\
\hline - Male & 77 & 52 & $67.5 \%$ & $44.7 \%$ & $27.7 \%$ & \\
\hline \multicolumn{7}{|l|}{ Ethnicity: } \\
\hline -White & 93 & 54 & $74.2 \%$ & $51.9 \%$ & $35.7 \%$ & 0.002 \\
\hline - Non-white & 19 & 17 & $52.6 \%$ & $21.1 \%$ & $7.0 \%$ & \\
\hline \multicolumn{7}{|l|}{ Age: } \\
\hline$-\leq 20$ years & 74 & 44 & $75.7 \%$ & $54.5 \%$ & $35.8 \%$ & 0.023 \\
\hline$->20$ years & 39 & 28 & $59.0 \%$ & $26.9 \%$ & $17.9 \%$ & \\
\hline \multicolumn{7}{|l|}{ Tumor Size: } \\
\hline - Small & 9 & 4 & $88.9 \%$ & $55.6 \%$ & $55.6 \%$ & 0.162 \\
\hline - Large & 69 & 47 & $65.2 \%$ & $43.1 \%$ & $24.1 \%$ & \\
\hline \multicolumn{7}{|l|}{$\begin{array}{l}\text { Tumor location } \\
\text { (tissue): }\end{array}$} \\
\hline - Bone & 66 & 45 & $74.2 \%$ & $42.6 \%$ & $26.5 \%$ & 0.602 \\
\hline - Soft tissues & 47 & 27 & $63.8 \%$ & $52.2 \%$ & $37.0 \%$ & \\
\hline \multicolumn{7}{|l|}{$\begin{array}{l}\text { Tumor location } \\
\text { (topography): }\end{array}$} \\
\hline - Axial & 59 & 38 & $67.8 \%$ & $41.8 \%$ & $32.2 \%$ & 0.614 \\
\hline - Peripheral & 54 & 34 & $72.2 \%$ & $50.4 \%$ & $31.7 \%$ & \\
\hline \multicolumn{7}{|l|}{$\begin{array}{l}\text { Disease extent at } \\
\text { diagnosis: }\end{array}$} \\
\hline - Localized & 54 & 27 & $79.6 \%$ & $61.7 \%$ & $43.1 \%$ & 0.003 \\
\hline - Metastatic & 55 & 42 & $60.0 \%$ & $29.0 \%$ & $20.1 \%$ & \\
\hline
\end{tabular}

\begin{tabular}{|c|c|c|c|c|}
\hline \multirow[t]{2}{*}{ Characteristic } & \multirow[t]{2}{*}{ HR } & \multicolumn{2}{|c|}{$95 \% \mathrm{Cl}$} & \multirow[t]{2}{*}{$p$-value } \\
\hline & & Inferior & Superior & \\
\hline \multicolumn{5}{|l|}{ MTAP: } \\
\hline - Loss of expression & 1.818 & 1.029 & 3.215 & 0.039 \\
\hline - Positive expression & 1 & - & - & \\
\hline \multicolumn{5}{|l|}{ Ethnicity: } \\
\hline -White & 1 & -- & - & 0.001 \\
\hline - Non-white & 3.401 & 1.643 & 7.037 & \\
\hline \multicolumn{5}{|l|}{ Age: } \\
\hline$-\leq 20$ years & 1 & 一- & - & 0.084 \\
\hline$->20$ years & 1.754 & 0.928 & 3.314 & \\
\hline \multicolumn{5}{|c|}{ Disease extent at diagnosis: } \\
\hline - Localized & 1 & 一 & - & 0.002 \\
\hline - Metastatic & 2.476 & 1.400 & 4.377 & \\
\hline
\end{tabular}




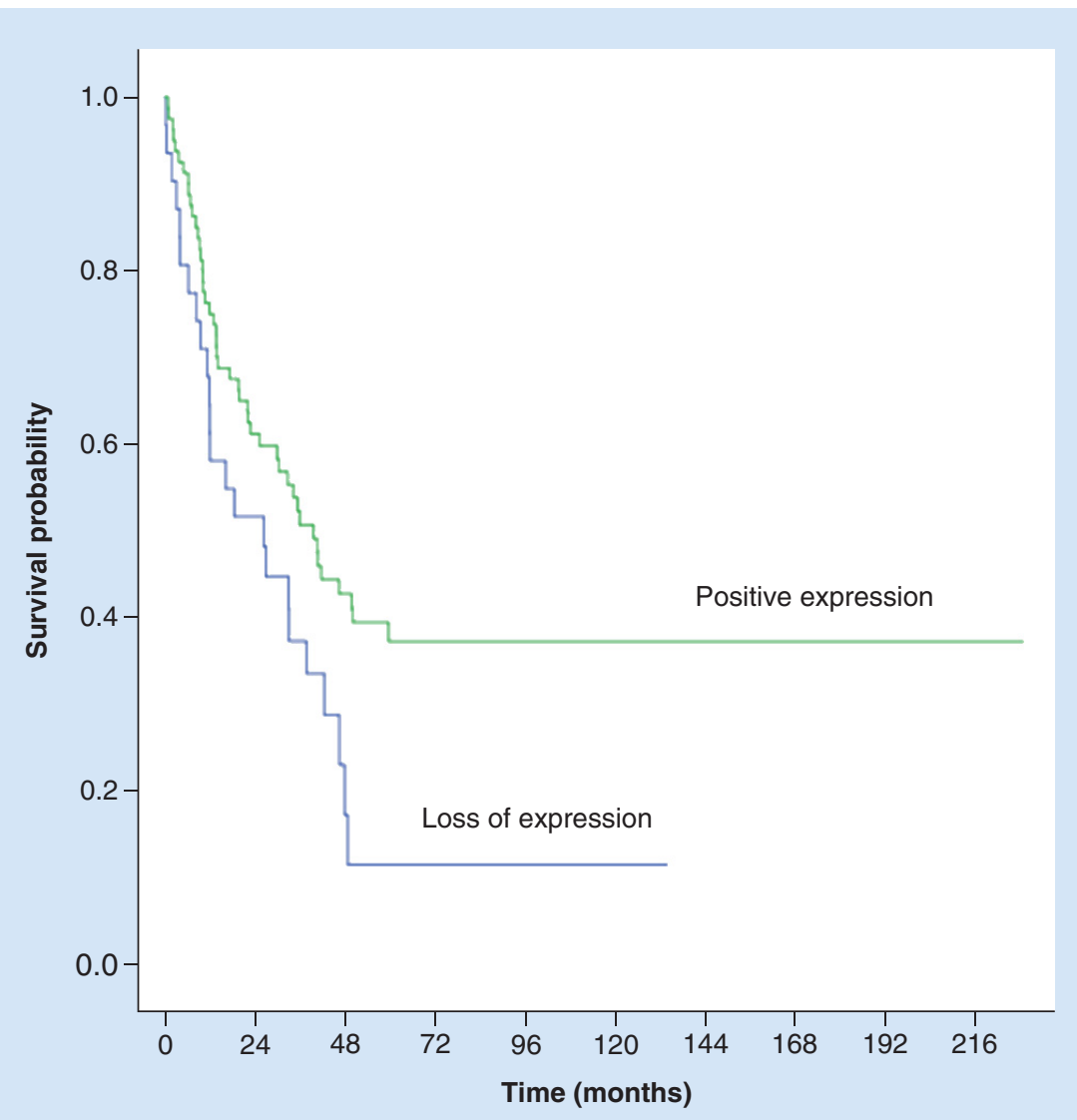

Figure 2. Kaplan-Meier overall survival curves according to methylthioadenosine phosphorylase expression

Table 5. Multivariate Cox regression model in metastatic and nonmetastatic patients.

\begin{tabular}{|c|c|c|c|c|c|}
\hline \multirow[t]{2}{*}{ Patients' group } & \multirow[t]{2}{*}{ MTAP } & \multirow[t]{2}{*}{ HR } & \multicolumn{2}{|c|}{$95 \% \mathrm{Cl}$} & \multirow[t]{2}{*}{ p-value } \\
\hline & & & Inferior & Superior & \\
\hline Metastatic & Loss of expression & 2.352 & 1.135 & 4.878 & 0.021 \\
\hline$(n=54)$ & Positive expression & 1 & - & - & \\
\hline Nonmetastatic & Loss of expression & 2.334 & 0.450 & 12.107 & 0.313 \\
\hline$(n=53)$ & Positive expression & 1 & - & - & \\
\hline
\end{tabular}

HR: Hazard ratio; MTAP: Methylthioadenosine phosphorylase.

ESFT are known to be more common in white patients when compared with patients from other ethnic groups, according to literature data [9,10,14,15]. Jawad et al. (2009) [14] in a retrospective analysis of 1631 cases from the population-based SEER (Surveillance, Epidemiology and End Results) program of the National Cancer Institute showed that Caucasians had a ninefold higher incidence of ESFT than African-Americans. In the present study, $83 \%$ of the patients $(n=93)$ were white, corroborating the data in the literature and presenting numbers that were practically identical to those of the first multicentric study of the Brazilian Group for Treatment of ESFT, by Brunetto et al. (2015) [10], which showed $82.9 \%$ of white patients in a total of 175 cases studied. Ethnicity was an independent prognostic factor in our study $(\mathrm{p}=0.003)$. Non-white patients had OS rates lower than those of white patients. In fact, non-white patients had a 3.4-fold higher risk of death when compared with white patients (95\% CI: 1.64-7.03). Worch et al. (2010) [15], in a large retrospective study of 1715 patients from the SEER database, showed that OS was significantly different according to the ethnicity/race of the patients, being lower in non-white patients, in agreement with our results. These differences in clinical outcomes may reflect socioeconomic and biological differences or a combination of both factors. It is known that the socioeconomic reality of the non-white population, especially black and indigenous, is extremely unfavorable in Brazil. The low socioeconomic status could 


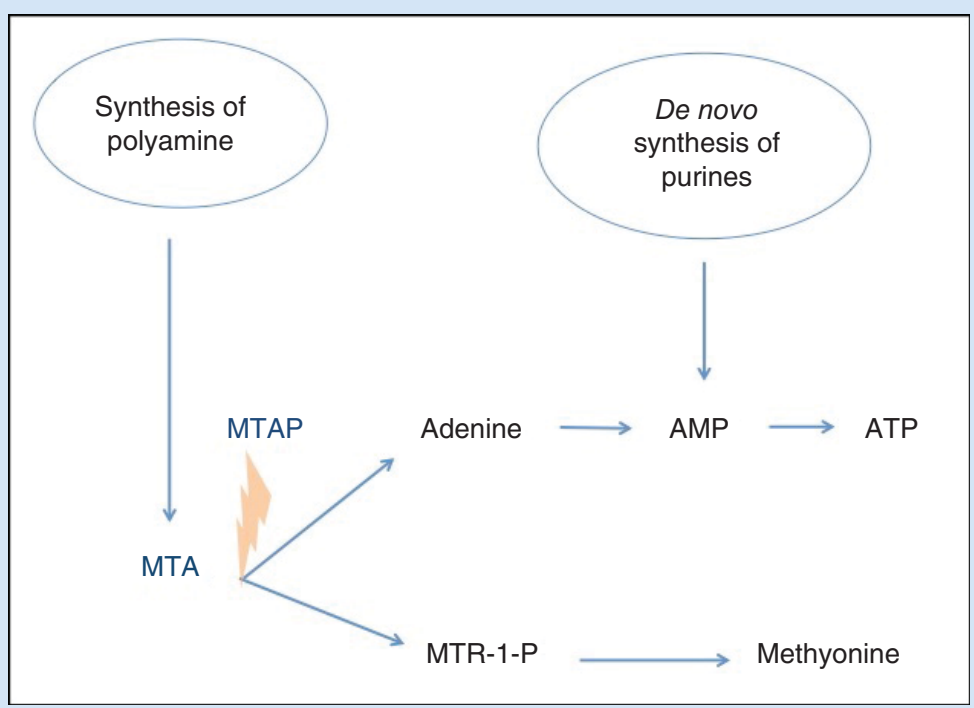

Figure 3. Methylthioadenosine phosphorylase metabolic pathway. In normal cells, MTAP cleaves MTA, a byproduct of polyamine biosynthesis, into adenine and MTR-1-P. In MTAP-deficient cells, however, the MTA is not cleaved and the salvage pathway for adenine and methionine is absent, with a stimulus for the de novo synthesis of purines. MTA: Methylthioadenosine; MTAP: Methylthioadenosine phosphorylase; MTR-1-P: 5-methylthioribose-1-phosphate.

limit or delay the access of these patients to the diagnosis and treatment, thus influencing their prognoses. Biological differences may include variations in tumor aggressiveness and host factors, such as genetic differences that would influence tumor metabolism and response to treatment. However, broader and more focused studies are needed to investigate these possible biological and genetic differences among ethnicities, as well as additional studies on disparities in health care access to assess the role of these factors in explaining the differences in the outcomes.

The MTAP biomarker showed loss of expression in $27.9 \%$ of the cases $(\mathrm{n}=31)$. To best of our knowledge, these findings have never been reported in ESFT. Besides, this is the first study to show the association of loss of MTAP expression with shorter OS in these patients.

$M T A P$ is expressed in all normal tissues, but is often deleted in some tumors, mainly in co-deletion with the $C D K N 2 A$ gene. Therefore, it is attributed a tumor suppression function to the MTAP gene [4,16]. In normal cells, the MTAP protein acts on the purine biosynthesis pathway, being important to the salvage process of adenine and methionine by cleaving the substrate $5^{\prime}$-deoxy-5'-Methylthioadenosine (MTA) generated during the biosynthesis of polyamines, and producing adenine and 5-methylthioribose-1-phosphate (MTR-1-P), which is later converted to methionine. This pathway is the only known source of free adenine in the cells (Figure 3).

Inactivation of MTAP and the loss of its expression in tumor cells are associated with the de novo synthesis of purines, which results in increased cell proliferation and, consequently, more aggressive tumor subtypes [4]. In the study by Su et al. (2014), loss of MTAP expression in a series of non-small-cell lung carcinomas, assessed by IHC, was independently associated with prognosis [3]. Also in line with our results, He et al. (2015) demonstrated that MTAP deficiency was correlated with a more aggressive phenotype of nasopharyngeal carcinoma and with shorter survival [17]. Recently, Nan et al. (2017) concluded that low MTAP expression was also associated with poorer prognosis in ovarian cancer [18]. In the present study, the ESFT patients with loss of MTAP expression had a 1.81-times greater risk of death when compared with patients with positive immunoexpression (95\% CI: 1.02-3.21). When the patients were classified into metastatic and nonmetastatic groups, loss of MTAP expression showed significant correlation with OS only in the metastatic group, in both univariate and multivariate analyses $(p=0.021)$. In fact, in the metastatic group, patients with MTAP loss were 2.35-times more likely to die rather than patients with MTAP positivity (95\% CI: 1.13-4.87). These results suggest that MTAP is a prognostic factor, particularly robust in advanced cases, probably due to the biological mechanisms of aggressiveness associated with its deficiency. However, apart from OS, our results did not demonstrate association of MTAP with any other clinical feature commonly related to tumor aggressiveness, such as tumor size and metastasis. We believe that the 
high number of patients with large tumors and metastases by the time of diagnosis in this series may have affected the statistical results and masked any possible relation of this marker with these variables. Besides, we cannot rule out the possibility of MTAP having an association with treatment response, which was not explored in this study. Therefore, further studies are warranted for extending and validating the present important findings.

The finding that the MTAP enzyme is generally absent in some types of cancer has led to the proposition of selective therapies, which utilizes gene deficiency to specifically target MTAP-negative tumor cells [19]. In this context, the analysis of MTAP expression in ESFT may contribute to the development and employment of new therapeutic strategies. The currently used protocols for chemotherapy in ESFT include five agents: vincristine, doxorubicin, cyclophosphamide, ifosfamide and etoposide (VDC/IE). Therefore, in addition to the significance in prognosis, MTAP could also have an important role as a target for molecular therapies to be developed. Also, MTAP may have a potential as a predictor of responsiveness to some available drugs. Recent researches involving other types of cancer, such as the study by De Oliveira et al. (2016) in breast cancer [4], have shown that tumor cells with loss of MTAP, due to gene deletion or methylation of the MTAP promoter, are more sensitive to purine synthesis inhibitor agents such as methotrexate, azathioprine and $5^{\prime}$-fluorouracil $\left(5^{\prime}-\mathrm{FU}\right)$ [1,2]. Despite these drugs did not appear to have a significant therapeutic activity against the ESFT in the past [20], and the relative success of current strategies in cases of localized disease, the possibility of using a combination of both schemes in MTAP-negative cases cannot be disregarded after prospective studies. Thus, a subset of patients with ESFT exhibiting loss of MTAP could potentially benefit from treatment with specific chemotherapy, especially when disease is disseminated and is not responding to conventional treatment.

\section{Conclusion}

With these evidences, it is demonstrated that the loss of MTAP expression may be useful as a predictor of worse prognosis in patients with ESFT. MTAP along with other biomarkers and clinical factors associated with the prognosis may aid in the elaboration of a future risk stratification for these tumors and even stimulate the development of specific targeted drugs, especially for advanced disease.

\section{Summary points}

- Ewing sarcoma family of tumors (ESFT) are neoplasms of primitive neuroectodermal cells with an aggressive biological behavior and poor outcomes.

- Survival rates for patients with metastatic or recurrent ESFT have not improved as much as rates for patients with localized disease during the last decades.

- Loss of methylthioadenosine phosphorylase (MTAP) expression is observed in approximately $28 \%$ of ESFT.

- Loss of MTAP expression is an independent prognostic factor associated with shorter overall survival in patients with ESFT.

- In MTAP-deficient cells, the salvage pathway for adenine and methionine is absent, causing a stimulus for the de novo synthesis of purines which is associated with increased tumor aggressiveness.

- The use of MTAP as a prognostic biomarker in ESFT may aid in the risk stratification of patients and stimulate the development of specific targeted drugs.

Financial \& competing interests disclosure

This study was partially supported by Fundação de Amparo à Pesquisa do Estado de São Paulo (FAPESP) and CNPq grants to RM Reis. WP Menezes is recipient of a FAPESP fellowship (2016/06833-2). The authors have no other relevant affiliations or financial involvement with any organization or entity with a financial interest in or financial conflict with the subject matter or materials discussed in the manuscript apart from those disclosed.

No writing assistance was utilized in the production of this manuscript

Ethical conduct of research

The study was conducted following national and institutional ethical policies and it was previously approved by the Barretos Cancer Hospital Ethical Committee (protocol 935/2015). Since it was classified as having minimal risk, ensuring confidentiality and not resulting in any clinical influences for the patients, the ethical committee waived the need for consent in this study. 


\section{References}

Papers of special note have been highlighted as: $\bullet \bullet$ of considerable interest

1. Bertino JR, Waud WR, Parker WB, Lubin M. Targeting tumors that lack methylthioadenosine phosphorylase (MTAP) activity: current strategies. Cancer Biol. Ther. 11(7), 627-632 (2011).

-. In this important review, the frequency of methylthioadenosine phosphorylase deficiency in solid tumors is presented and the strategies to target such deficient cells are discussed in a very instructive manner.

2. Lubin M, Lubin A. Selective killing of tumors deficient in methylthioadenosine phosphorylase: a novel strategy. PLoS ONE 4(5), e5735 (2009).

3. Su CY, Chang YC, Chan YC et al. MTAP is an independent prognosis marker and the concordant loss of MTAP and p16 expression predicts short survival in non-small cell lung cancer patients. Eur. J. Surg. Oncol. 40(9), 1143-1150 (2014).

4. De Oliveira SF, Ganzinelli M, Chila R et al. Characterization of MTAP gene expression in breast cancer patients and cell lines. PLoS ONE 11(1), e0145647 (2016).

5. Becker AP, Scapulatempo-Neto C, Menezes WP et al. Expression of methylthioadenosine phosphorylase (MTAP) in pilocytic astrocytomas. Pathobiology 82(2), 84-89 (2015).

6. De Alava E, Lessnick SL, Sorensen PH. Ewing Sarcoma. In: WHO Classification of Tumours of Soft Tissue and Bone. Fletcher DM, Bridge JA, Hogendoorn PCW, Mertens F (Eds). IARC Lyon, France (2013).

7. Kim SK, Park YK. Ewing sarcoma: a chronicle of molecular pathogenesis. Hum. Pathol. 55, 91-100 (2016).

-• Summarizes a chronicle of molecular findings of Ewing sarcoma family of tumors including the most recent advances.

8. Hamilton SN, Carlson R, Hasan H, Rassekh SR, Goddard K. Long-term outcomes and complications in pediatric Ewing sarcoma. Am. J. Clin. Oncol. 40(4), 423-428 (2015).

9. Rodriguez-Galindo C, Liu T, Krasin MJ et al. Analysis of prognostic factors in Ewing sarcoma family of tumors: review of St. Jude Children's Research Hospital studies. Cancer 110(2), 375-384 (2007).

10. Brunetto AL, Castillo LA, Petrilli AS et al. Carboplatin in the treatment of Ewing sarcoma: results of the first Brazilian collaborative study group for Ewing sarcoma family tumors-EWING1. Pediatr. Blood Cancer 62(10), 1747-1753 (2015).

11. Cash T, Mcilvaine E, Krailo MD et al. Comparison of clinical features and outcomes in patients with extraskeletal versus skeletal localized Ewing sarcoma: A report from the Children’s Oncology Group. Pediatr. Blood Cancer 63(10), 1771-1779 (2016).

12. Lee J, Hoang BH, Ziogas A, Zell JA. Analysis of prognostic factors in Ewing sarcoma using a population-based cancer registry. Cancer 116(8), 1964-1973 (2010).

13. Carneseca EC, Mauad EC, De Araujo MA, Dalbo RM, Longatto Filho A, Vazquez Vde L. The Hospital de Cancer de Barretos Registry: an analysis of cancer survival at a single institution in Brazil over a 10-year period. BMC Res. Notes 6, 141 (2013).

14. Jawad MU, Cheung MC, Min ES, Schneiderbauer MM, Koniaris LG, Scully SP. Ewing sarcoma demonstrates racial disparities in incidence-related and sex-related differences in outcome: an analysis of 1631 cases from the SEER database, 1973-2005.

Cancer 115(15), 3526-3536 (2009)

15. Worch J, Matthay KK, Neuhaus J, Goldsby R, Dubois SG. Ethnic and racial differences in patients with Ewing sarcoma. Cancer 116(4), 983-988 (2010).

16. Kim J, Kim MA, Min SY, Jee CD, Lee HE, Kim WH. Downregulation of methylthioadenosin phosphorylase by homozygous deletion in gastric carcinoma. Genes Chromosomes Cancer 50(6), 421-433 (2011).

17. He HL, Lee YE, Shiue YL, Lee SW, Chen TJ, Li CF. Characterization and prognostic significance of methylthioadenosine phosphorylase deficiency in nasopharyngeal carcinoma. Medicine (Baltimore) 94(49), e2271 (2015).

18. Nan DF, Xihan Y, Liyuan D, Luwei F, Yongzhi W et al. MTAP deficiency is associated with an unfavourable prognosis and platinum resistance in ovarian cancer. Int. J. Clin. Exp. Pathol. 10(2), 1122-1130 (2017).

19. Kadariya Y, Tang B, Myers CB, Fukui J, Peterson JR, Kruger WD. Chemical genetic screening for compounds that preferentially inhibit growth of methylthioadenosine phosphorylase (MTAP)-deficient Saccharomyces cerevisiae. J. Biomol. Screen. 16(1), 44-52 (2011).

20. Balis FM, Gillespie A, Belasco J et al. Phase II trial of sequential methotrexate and 5-fluorouracil with leucovorin in children with sarcomas. Invest. New Drugs 8(2), 181-182 (1990). 